\title{
Scattering in a Magnetic Field
}

\author{
David C. Carey \\ Fermi National Accelerator Laboratory \\ Batavia, Ill. 60563 \\ 16 August 2002
}




\section{Introduction}

The fixed target program at Fermilab has come to an end. New projects are in the planning stage. Among them is a muon storage ring.

Up to the present, all storage rings in high-energy physics have carried stable particles, namely the electron and proton and their antiparticles. The muon is unstable and decays with a mean lifetime of $2.0 \times 10^{-6} \mathrm{sec}$. Two types of cooling have been used in the past. One is stochastic cooling where an electrode is used to detect the positions of the particles and send a signal to another position across the ring. Through successive applications of this technique, the phase space is ultimately greatly reduced and beams can be made to collide with a useful event rate.

The second type of cooling is electron cooling. Here protons and electrons are made to travel together for a short distance. Equipartition causes transfer of transverse energy of the protons to that of the electrons.

Neither of these methods is fast enough to allow acceleration of a sufficient number of muons up to maximum energy before they decay. A new method known as ionization cooling has been proposed.[1] The muons are cooled by passing them through a container of liquid hydrogen. The energy loss reduces both transverse and longitudinal momentum. The longitudinal momentum is restored with RF cavities. The net result is to maintain the longitudinal momentum while cooling the transverse momentum.

To minimize the total travel distance of the muons the liquid hydrogen is placed inside the focusing solenoids. The question arises as to whether the presence of the solenoids influences the phase space occupied by the muons. After the muon scatters it has transverse momentum. In a constant longitudinal magnetic field the trajectory wraps around the field lines and coincides in momentum and position with a particle which scatters one cycle later.

Here we calculate the change in emittance for both a drift space and a solenoid. We find that the presence of the solenoid does cause a reduction in phase space. Shown below are

both a derivation of the behavior of the muon phase space and a plot showing the strength of the effect described. 


\section{Scattering in a Magnetic Field}

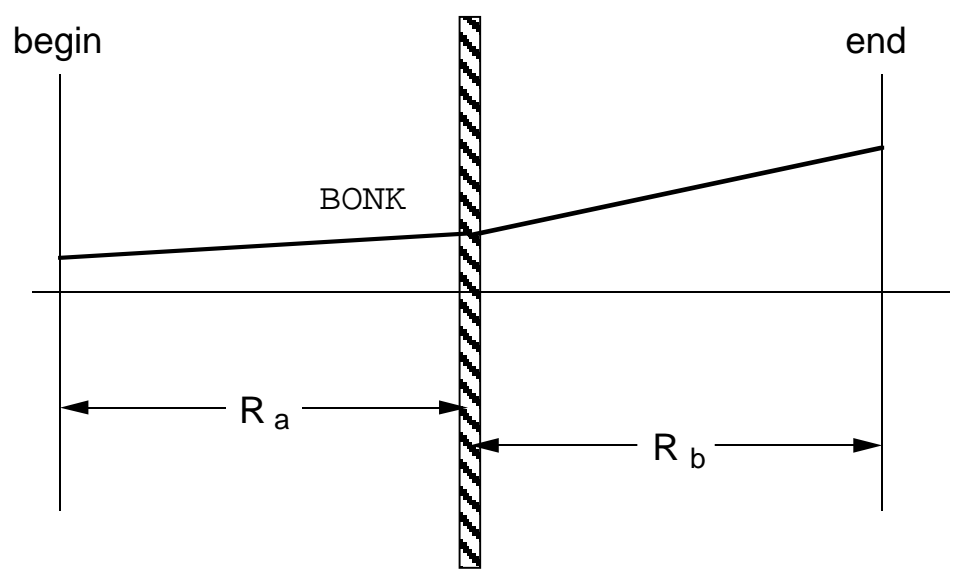

Figure 1: Scattering of a muon by a slice of liquid hydrogen.

\section{Multiple Scattering}

The rms scattering angle of a charged particle passing through a section of material medium, in a single transverse plane, is given by the equation[2]:

$$
\theta_{0}=\frac{13.6 \mathrm{Mev}}{\beta c p} z \sqrt{\frac{x}{X_{0}}}\left[1+0.038 \log \left(\frac{x}{X_{0}}\right)\right]
$$

Here $p$ is the muon momentum and $\beta c$ is the velocity. The letter $z$ is the charge number of the particle. For a muon, this number is, of course, 1.0. The thickness of the slab of material is $x$, and $X_{0}$ is the radiation length of the material.

To answer our question about the wrapping up of phase space we shall make a few approximations. Some of these approximations are necessary. However, their effect is small enough so as not to seriously change the answer to the question. Instead, by simplifying 
some part of the derivation we add greater clarity to the question about the reduction of phase space.

First we ignore any energy loss. The distance a muon can travel in material will be much longer than a wave length of magnetic focusing in a solenoid. Also it is difficult to calculate analytically the trajectory of a particle in a solenoid when the magnitude of the momentum is continuously changing. We also ignore any energy dependence of the rms width of the scattering on momentum.

Secondly, the value of $\theta_{0}$ derived is from a fit to a Moliere distribution. It is not the value that would be obtained from a direct fit to a Gaussian. We delete the term from equation (1) containing the logarithm. Then the effect of two scatters would be to add in quadrature, making the mathematics much simpler.

Finally, we assume that the magnetic field in the solenoid is uniform. As stated above, the equations of motion are simplified, since the projection of the motion on a plane perpendicular to the axis is a circle. If we restrict ourselves to first order, there are no singularities in the end fields. We can integrate right through the ends without any sudden discontinuities.

\section{The Effect of Scattering on the Beam Matrix}

From equation (1) we can determine that the effect on the beam (sigma) matrix at the point of scatter is given by [3]:

$$
\Delta \sigma_{x x}(s)=\Delta \sigma_{y y}(s)=K \Delta z
$$

The expressions $\Delta \sigma_{x x}(s)$ and $\Delta \sigma_{y y}(s)$ can be taken to be the changes in the second moments of the phase space distribution. The subscripts $x$ and $y$ represent the coordinates in the transverse configuration space.

Here we use the argument "s" to indicate the point of scatter. We shall also use the argument "b" and "e" of the sigma matrix to identify the beginning and end of the system respectively. The indices on the $R$ and $\sigma$ matrices correspond to a six-dimension vector of kinematic quantities. The vector is $\left(x, x^{\prime}, y, y, \ell, \delta\right)$. In our particular application, only the first four components are significant. 
Transforming the sigma matrix to the end of the system, we get:

$$
\Delta \sigma_{i j}(e)=K R_{i 2}(b) R_{j 2}(b) \Delta z+K R_{i 4}(b) R_{j 4}(b) \Delta z
$$

We break the total system up into two steps. The first step (a) is from the beginning of the system to the point of scatter. The second step (b) is from the point of scatter to the end of the system. The total transfer matrix is then:

$$
R(t)=R(b) R(a)
$$

or

$$
R(b)=R(t) R(a)^{-1}
$$

Substituting for $R(b)$ we get

$$
\begin{aligned}
\Delta \sigma_{i j}(e) & =K \sum_{k} \sum_{\ell} R_{i k}(t) R_{k 2}^{-1}(a) R_{j \ell}(t) R_{\ell 2}^{-1}(a) \Delta \\
& +K \sum_{k} \sum_{\ell} R_{i k}(t) R_{k 4}^{-1}(a) R_{j \ell}(t) R_{\ell 4}^{-1}(a) \Delta z
\end{aligned}
$$

Integrating

$$
\begin{aligned}
& \Delta \sigma_{i j}(e)=\sum_{k} \sum_{\ell} R_{i k}(t) R_{j \ell}(t) \times \\
& \quad\left[\int R_{k 2}^{-1}(a) R_{\ell 2}^{-1}(a)+\int R_{k 4}^{-1}(a) R_{\ell 4}^{-1}(a)\right] K d z
\end{aligned}
$$


The equation is for the components of the beam matrix at the end of the system. The equation for the beam matrix at the beginning of the system is obtained by omitting the two summations and the two factors of $R(t)$ on the right side of the equation. Since we will eventually be taking determinants, this omission will not change the answer.

$$
\Delta \sigma_{i j}(b)=\left[\int R_{k 2}^{-1}(a) R_{\ell 2}^{-1}(a)+\int R_{k 4}^{-1}(a) R_{\ell 4}^{-1}(a)\right] K d z
$$

\section{Factorized Transfer Matrices}

Taking the inverse or determinant of a four-by-four matrix is a procedure fraught with peril, and guaranteed to produce more errors than correct calculations. Fortunately in this case the total transfer matrix factors into a product of matrices. Each of these matrices is much simpler than the product matrix.

From the analytic form of the product of matrices, we can see that one matrix represents a geometric rotation of two planes[4][5]. The rotating frame is known as the Larmor frame.

The dynamics all take place in the rotating plane. The motions in the two transverse planes are independent. They consist of harmonic oscillations in each of the two planes.

If the magnetic field is not uniform the dynamic motion in the rotated plane is a general matrix with cosinelike and sinelike functions executing a harmonic motion of changing period. Since the Larmor frame is a geometric rotation, the components of the transformation matrix 
can be given directly in terms of trigonometric functions.

\section{Non-Uniform Field}

$$
R=\left(\begin{array}{cccc}
c_{u} & s_{y} & 0 & 0 \\
c_{u}^{\prime} & s_{u}^{\prime} & 0 & 0 \\
0 & 0 & c_{v} & s_{v} \\
0 & 0 & c_{v}^{\prime} & s_{v}^{\prime}
\end{array}\right)\left(\begin{array}{cccc}
\cos \beta & 0 & \sin \beta & 0 \\
0 & \cos \beta & 0 & \sin \beta \\
-\sin \beta & 0 & \cos \beta & 0 \\
0 & -\sin \beta & 0 & \cos \beta
\end{array}\right)
$$

\section{$\underline{\text { Uniform Field }}$}

$$
R=\left(\begin{array}{cccc}
\cos k z & \frac{1}{k} \sin k z & 0 & 0 \\
-k \sin k z & \cos k z & 0 & 0 \\
0 & 0 & \cos k z & \frac{1}{k} \sin k z \\
0 & 0 & -k \sin k z & \cos k z
\end{array}\right)\left(\begin{array}{cccc}
\cos k z & 0 & \sin k z & 0 \\
0 & \cos k z & 0 & \sin k z \\
-\sin k z & 0 & \cos k z & 0 \\
0 & -\sin k z & 0 & \cos k z
\end{array}\right)
$$


Inverse Matrix

$R=\left(\begin{array}{cccc}\cos k z & -\frac{1}{k} \sin k z & 0 & 0 \\ k \sin k z & \cos k z & 0 & 0 \\ 0 & 0 & \cos k z & -\frac{1}{k} \sin k z \\ 0 & 0 & k \sin k z & \cos k z\end{array}\right)\left(\begin{array}{cccc}\cos k z & 0 & -\sin k z & 0 \\ 0 & \cos k z & 0 & -\sin k z \\ \sin k z & 0 & \cos k z & 0 \\ 0 & \sin k z & 0 & \cos k z\end{array}\right)$

\section{Integrated Product of Inverse Matrices}

\section{Drift Spaces}

If we do the integration equation for a drift space, we derive:

$$
\begin{gathered}
\int \operatorname{sum}=\left(\begin{array}{cccc}
\frac{1}{3} L^{3} & -\frac{1}{2} L^{2} & 0 & 0 \\
-\frac{1}{2} L^{2} & L & 0 & 0 \\
0 & 0 & \frac{1}{3} L^{3} & -\frac{1}{2} L^{2} \\
0 & 0 & -\frac{1}{2} L^{2} & L
\end{array}\right) \\
\text { Determinant }=\frac{1}{144} L^{8}
\end{gathered}
$$


We drop the factor $K$ as we shall be taking the ratio of determinants and factors of $K$ will cancel.

For a single transverse plane:

$$
\text { Determinant }=\frac{1}{12} L^{4}
$$

\section{Solenoids}

Evaluating the matrix for a solenoid takes a little more effort. Here we let the program MATHEMATICA[6] do the manipulation. We then arrive at

$$
\int \operatorname{sum}=\left(\begin{array}{cccc}
\frac{1}{2 k^{2}}\left(L-\frac{C S}{k}\right) & -\frac{S^{2}}{2 k^{2}} & 0 & 0 \\
-\frac{S^{2}}{2 k^{2}} & \frac{1}{2}\left(L+\frac{C S}{k}\right) & 0 & 0 \\
0 & 0 & \frac{1}{2 k^{2}}\left(L-\frac{C S}{k}\right) & -\frac{S^{2}}{2 k^{2}} \\
0 & 0 & -\frac{S^{2}}{2 k^{2}} & \frac{1}{2}\left(L+\frac{C S}{k}\right)
\end{array}\right)
$$

where

$$
\begin{aligned}
C & =\cos k L \\
S & =\sin k L \\
k & =\frac{q B}{2 p}
\end{aligned}
$$


For a single transverse plane:

$$
\text { Determinant }=\frac{1}{4 k^{2}}\left(L^{2}-\frac{S^{2}}{k^{2}}\right)
$$

\section{Ratio of Emittances}

The horizontal and vertical emittances in terms of elements of the beam matrix are given by:

$$
\begin{aligned}
\epsilon_{H}^{2} & =\sigma_{11} \sigma_{22}-\sigma_{21}^{2} \\
\epsilon_{V}^{2} & =\sigma_{33} \sigma_{44}-\sigma_{43}^{2}
\end{aligned}
$$

From this we can derive the ratio of emittances in a single transverse plane: $\epsilon_{S}$ for a solenoid, and $\epsilon_{D}$ for a drift.

$$
\text { Ratio }=\frac{\epsilon_{S}}{\epsilon_{D}}=\sqrt{\frac{3}{\phi^{2}}\left(1-\frac{S^{2}}{\phi^{2}}\right)}
$$

where the Larmor phase $\phi$ is given by: 


$$
\phi=k L
$$

and

$$
S=\sin \phi
$$

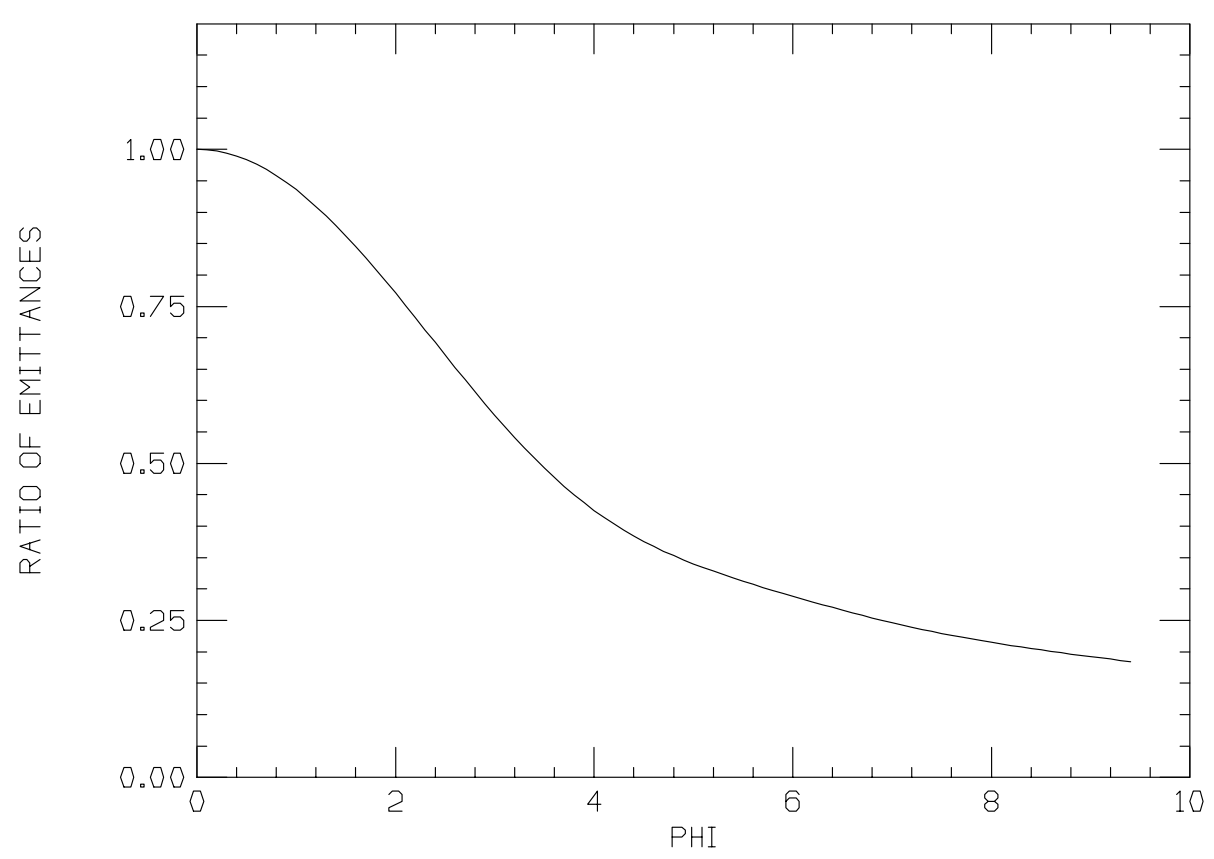

Figure 2: The ratio of horizontal emittances for solenoids and drift spaces.

\section{Acknowledgements}

The author wishes to express appreciation for suggestions and advice to Valeri Belbekov, Mike Syphers, Michael Kriss, Norman Gelfand, and Paul LeBrun. 


\section{References}

[1] Ankenbrandt et. al., Ionization Cooling Research and Development Program for a High Luminosity M Proposal, April 1998.

[2] Review of Particle Physics, The European Physics Journal 15, 1-4, 2000.

[3] David C. Carey, The Optics of Charged Particle Beams, Harwood Academic Publishers, 1987.

[4] Martin Reiser, Theory and Design of Charged Particle Beams, John Wiley \& Sons, 1994.

[5] Klaus Halbach, private communication.

[6] Wolfram, Stephen, The MATHEMATICA BOOK, Wolfram Media and Cambridge University Press, 1991. 